\title{
金属有机框架(MOFs)材料在锂离子电池及锂金属电池电解液中的应用
}

\author{
常智 $b, c$ 乔羽 $b$ 杨慧军 $b, c$ 邓瀚 $b, c$ 朱星宇 $b, c$ \\ 何平 $a$ 周豪慎 $*, a, b, c$ \\ ( ${ }^{a}$ 南京大学储能材料与技术中心 现代工程与应用科学学院 固体微结构国家重点实验室 \\ 人工微结构科学与技术协同创新中心 中国南京 210093) \\ ( ${ }^{b}$ 产业技术综合研究所(AIST) 能源技术研究所 筑波 305-8568 日本) \\ ( ${ }^{c}$ 日本国立筑波大学(University of Tsukuba) 系统与信息工程研究生院 筑波 305-8573 日本)
}

\begin{abstract}
摘要 总结了金属有机框架(MOFs)材料在锂离子电池电解液中的研究进展. 通过归纳锂离子电池长期存在的一些缺 陷，随后将 MOFs 材料作为离子篎、人造负极保护层、准固态电解质以及用来调节电解液构型，使得锂离子电池的性 能得到显著提升. 最后, 基于 MOFs 材料本身的特性, 还对 MOFs 材料在电化学储能领域中的后续应用进行了合理地前 瞻性展望.
\end{abstract}

关键词 金属有机框架(MOFs); 锂离子电池; 离子篮; 负极保护; 准固态电解质

\section{Applications of Metal-organic Frameworks (MOFs) Materials in Lithium-ion Battery/Lithium-metal Battery Electrolytes}
Zhi Chang ${ }^{b, c}$
Yu Qiao ${ }^{b}$
Huijun Yang ${ }^{b, c}$
Han Deng ${ }^{b, c}$
Xingyu $\mathrm{Zhu}^{b, c}$
Ping $\mathrm{He}^{a}$ Haoshen Zhou ${ }^{*, a, b, c}$

$\left({ }^{a}\right.$ Center of Energy Storage Materials \& Technology, College of Engineering and Applied Sciences, National Laboratory of Solid State Microstructures, and Collaborative Innovation Center of Advanced Microstructures, Nanjing University, Nanjing 210093, China)

( ${ }^{b}$ Energy Technology Research Institute, National Institute of Advanced Industrial Science and Technology (AIST), Tsukuba 305-8568, Japan)

( ${ }^{c}$ Graduate School of System and Information Engineering, University of Tsukuba, 1-1-1, Tennoudai, Tsukuba 305-8573, Japan)

\begin{abstract}
This perspective summarizes the research progress of metal-organic frameworks (MOFs) materials used in lithium-ion battery/lithium-metal electrolytes. By summarizing several long-standing defects of lithium-ion batteries/lithiummetal batteries, MOFs materials are subsequently used as ion sieves, artificial electrode protective layers, quasi-solid electrolytes and used to adjust electrolytes' configuration, the performance of batteries has been significantly improved. Finally, based on the characteristics of MOFs materials themselves, this perspective also makes a reasonable forward-looking outlook on the subsequent applications of MOFs materials in the field of electrochemical energy storage.

Keywords metal-organic framework (MOF); lithium-ion battery; ion sieves; negative electrode protection; quasi-solid electrolyte
\end{abstract}

\section{1 引言}

高速发展的现代社会导致的日渐严重的环境污染 以及能源短缺问题对新型能源存储与转化技术的发展 提出更高的要求 ${ }^{[1-2]}$. 锂离子电池作为一种高效的清洁 能源存储设备, 备受科研工作者关注. 得益于广大科研 工作者的不解努力, 锂离子电池已经实现了商业化, 并 在日常生活中得到广泛地使用. 然而, 日常使用的锂离 子电池尚存在如能量密度低、使用寿命短以及安全性能
差等诸多缺陷 ${ }^{[3]}$. 这大大限制了其在如电动车等对能量 密度、循环寿命以及安全性具有较高要求的前沿领域中 的进一步应用 ${ }^{[4-5]}$.

为了使锂离子电池能够满足上述前沿领域对能量 密度、循环使用寿命以及安全性的要求, 广大科研工作 者在诸多方面进行了大量的尝试和努力. 例如, 在开发 功能性电解液/添加剂/锂盐方面, 科学家们在经过大量 地尝试和努力之后, 获得了能量密度、循环寿命和安全

* E-mail: hszhou@nju.edu.cn

Received September 23, 2020; published November 6, 2020.

Project supported by the National Key Research and Development Program of China (No. 2016YFB0100203) and the National Natural Science Foundation of China (Nos. 21673166, 21633003, u1801252).

项目受国家重点研究发展计划(No. 2016YFB0100203)和国家自然科学基金(Nos. 21673166, 21633003, u1801252)资助. 
性均大大提升的锂离子电池/锂金属电池 ${ }^{[6-9]}$. 关于负极 保护的一些开创性工作也不断地被报道出来 ${ }^{[10-13]}$. 开发 高电压正极材料, 由于可以有效提高电池的能量密度, 也受到了很多科研工作者的重视 ${ }^{[14]}$. 近年来科研工作 者将金属有机框架(Metal-Organic Frameworks, MOFs) 材料引入到锂离子电池中, 用以解决锂离子电池中一些 长期存在的问题. MOFs 材料是一类由金属离子和有机 配体经过配位形成的多孔材料. 得益于广泛存在的金属 盐离子以及多种具有不同化学结构的有机配体, 科学家 们合成了诸多具有不同微纳结构以及表界面性质的 MOFs 材料. 鉴于其丰富的金属核心和较高的比表面积, MOFs 材料在许多科研领域内得到广泛研究 ${ }^{[15-20]}$. 例如, 科研工作者已将各种具有不同结构和功能的 MOFs 材料 应用于气体的分离、吸附及存储 ${ }^{[21-23]}$. 得益于其特殊的 多孔结构和均匀分布的金属核心位, MOFs 材料还被作 为催化剂以及催化剂载体应用于各类催化反应中 ${ }^{[24-26]}$. 近年来, MOFs 材料开始逐渐被引入到锂离子电池领域, 并显示出重要的科研和应用价值 ${ }^{[27-28]}$. 本文主要从将 MOFs 材料作为离子耖、金属负极保护层及准固态电解 质等几个方面的研究现状进行总结, 并对 MOFs 材料在 锂离子电池领域的潜在应用前景进行了前瞻性地展望.

\section{MOFs 材料在锂离子电池领域的最新进展}

通常情况下, 并不是所有的 MOFs 材料都可以被应 用于锂离子电池/锂金属电池之中. 考虑到 MOFs 材料 的化学稳定性和电化学稳定性, 只有一部分具有特殊性 质的 MOFs 材料能被应用到锂离子电池/锂金属电池体 系之中. 例如, 铜基 MOFs, 锆基 MOFs, 铁基 MOFs 以 及铝基 MOFs 材料 ${ }^{[15-16]}$, 由于本身结构稳定, 在酸碱体 系里也有优异的稳定性, 因而经常作为功能性的 MOFs
而被应用于锂离子电池/锂金属电池体系之中.

\subsection{MOFs 材料用作离子穊}

能源需求的不断增长推动了储能技术的发展. 科研 工作者致力于研发出能够超越锂离子电池的新型储能 电池体系, 并获得更高的能量密度并降低成本. 由于理 论比容量高 $\left(1675 \mathrm{mAh} \cdot \mathrm{g}^{-1}\right)$ 和自然储备丰富, 以硫作为 正极材料的锂硫电池被视为最具希望的下一代储能电 池体系 ${ }^{[29-32]}$. 但是, 锂硫电池的发展由于充放电产物多 硫化锂的穿梭效应的存在, 而存在严重的容量衰减和寿 命减少等显著问题. 为了解决这一难题，科研工作者将 单质硫填入各种具有不同结构和表面性质的导电基底 材料中, 以期在某种程度上减缓多硫化锂的穿梭效

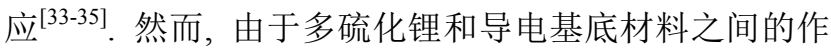
用并不牢固, 穿梭效应仍然存在 ${ }^{[36]}$. 所以, 迄今为止, 通过此类方法获得的锂硫电池的循环稳定性仍远未达 到预期的令人满意的程度.

2016 年, Bai 等 ${ }^{[37]}$ 首次将 MOF (Copper benzene1,3,5-tricarboxylate, 即 $\mathrm{Cu}_{3}(\mathrm{BTC})_{2}$, 简写成 $\mathrm{CuBTC}$ )创造 性地作为多硫化锂分子篎(图 $1 \mathrm{a}$ 和 $1 \mathrm{~b}$ ), 引入锂硫电池体 系之中. 得益于 CuBTC MOF 的狭小孔道 $(0.9 \mathrm{~nm})$, 具有 较大体积的多硫化锂 $(1.3 \mathrm{~nm})$ 被阻挡在正极区域内. 同 时, 该 CuBTC MOF 的孔道能够允许锂离子的自由传输. 鉴于此, 多硫化锂被有效地限制在正极区域内, 锂硫电 池常见的由多硫化锂穿梭导致的容量衰减被大大抑制. 如图 1c 所示, 使用 CuBTC MOF 作为离子篎的锂硫电池 展示出优异的电化学循环稳定性和容量保持率 $1 \mathrm{C}$ 倍率 下, 1500 圈, 71\%). Zang 等 ${ }^{[38]}$ 在 2018 年用一种二维导电 层状 $\mathrm{Ni}_{3}(\mathrm{HITP})_{2} \mathrm{MOF}$ 制备出无裂纹的 MOF 离子篮, 用 于抑制锂硫电池中的多硫化锂穿梭(图 1d 和 1e). 使用此 MOF 离子篮的锂硫电池在高载量的情况下, 获
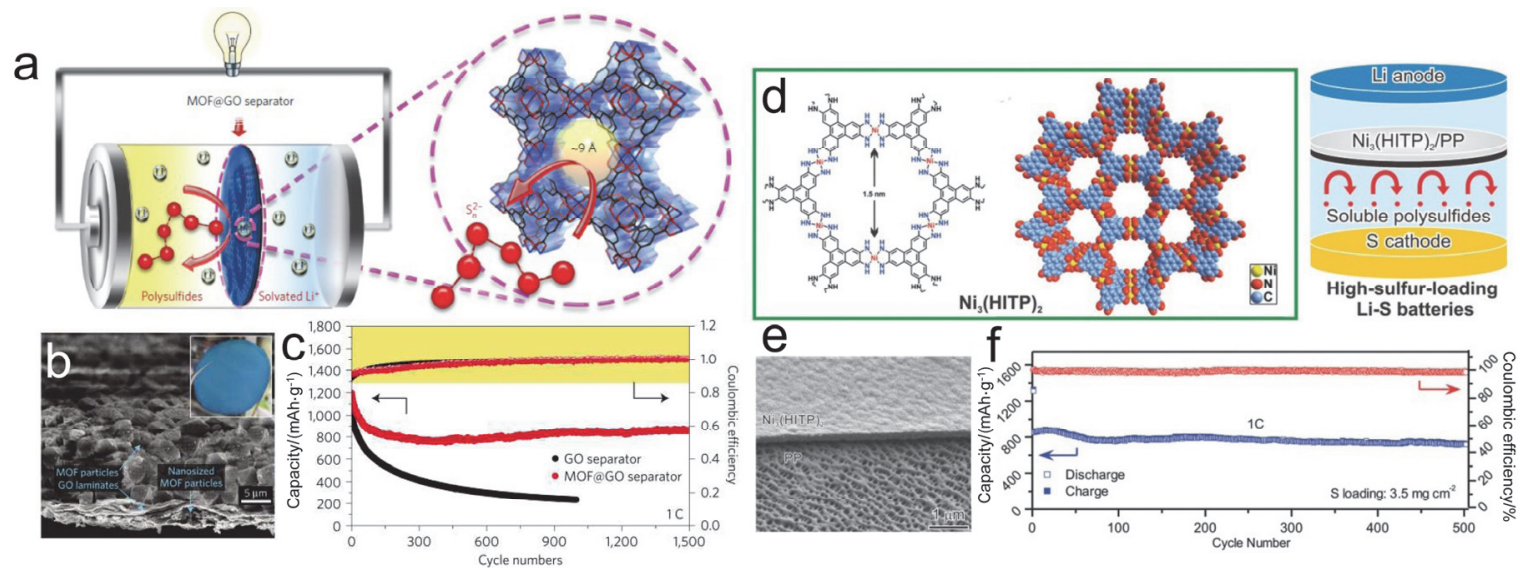

图 1 金属有机框架作为离子篮提升锂-硫电池的电化学性能. $(\mathrm{a} \sim \mathrm{c}) \mathrm{CuBTC}$ MOF 作为锂硫电池隔膜抑制多硫化锂穿梭, 提高锂-硫电池电化学性 能 ${ }^{[37}$. 经参考文献[37]许可转载, 版权 2016 自然出版集团. $(\mathrm{d} \sim \mathrm{f})$ 二维层状导电 $\mathrm{Ni}_{3}(\mathrm{HITP})_{2} \mathrm{MOF}$ 作为多硫化锂分子篮提升锂 - 硫电池循环稳定 性 ${ }^{[38]}$. 经参考文献[38]许可转载, 版权 2018 Wiley-VCH.

Figure 1 Metal-organic frameworks (MOFs) act as an ion sieves to improve the electrochemical performance of lithium-sulfur $(\mathrm{Li}-\mathrm{S})$ batteries. (a $\sim \mathrm{c})$ CuBTC MOF used as a Li-S battery separator to inhibit lithium polysulfide shuttling and improve the electrochemical performance of Li-S batteries ${ }^{[37]}$. Reprinted with permission from ref. [37], copyright 2016 Nature Publishing Group. (d f) Two-dimensional layered conductive $\mathrm{Ni}_{3}(\mathrm{HITP})_{2} \mathrm{MOF}$ is used as a lithium polysulfide molecular sieve to improve the cycle stability of Li-S batteries ${ }^{[38]}$. Reprinted with permission from ref. [38], copyright 2018 Wiley- $\mathrm{VCH}$. 

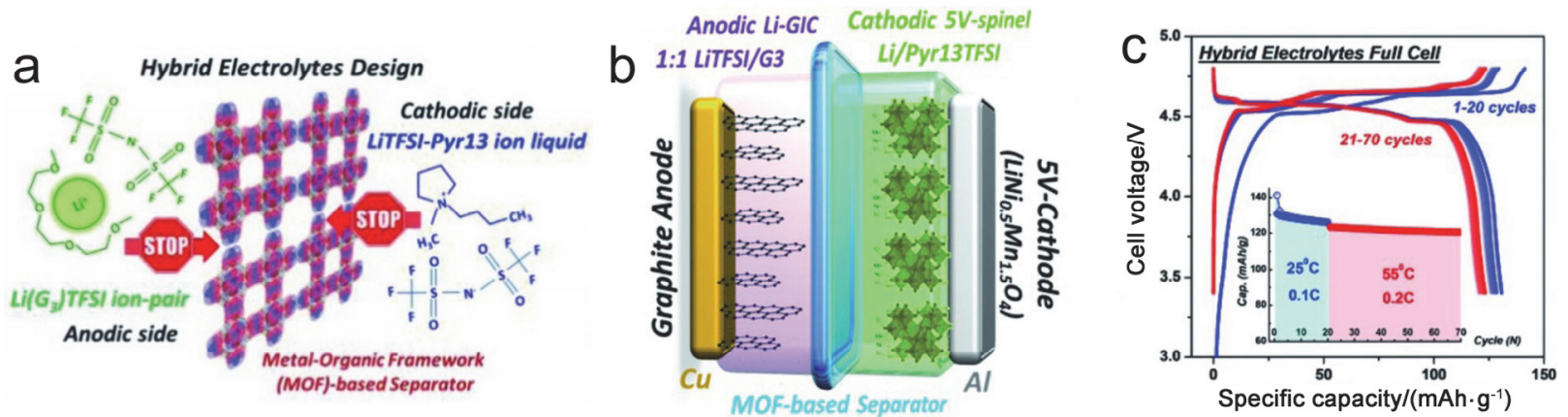

图 2 金属有机框架作为离子篮提升锂离子电池的电化学性能. (a c c CuBTC MOF 作为隔层, 分隔两种不同的电解液, 抑制电解液的相互穿梭, 实现组合电解液的电池设计, 最终提升 LNMO//Graphite 电池的电化学性能 ${ }^{[41]}$. 经参考文献[41]许可转载, 版权 2018 Wiley-VCH.

Figure 2 MOF acts as an ion sieves to improve the electrochemical performance of lithium ion batteries. $(\mathrm{a} \sim \mathrm{c}) \mathrm{CuBTC}$ MOF is used as a separator to separate two different electrolytes, inhibited the mutual shuttle of electrolytes, realized the battery design of combined electrolytes, and finally improved the electrochemical performance of LNMO//Graphite battery ${ }^{[41]}$. Reprinted with permission from ref. [41], copyright 2018 Wiley-VCH.

得优异的电化学性能(500 圈后, 容量保持在 $716 \mathrm{mAh}$ $\mathrm{g}^{-1}$, 保持率为 $84.1 \%$ ).

\subsection{MOFs 材料用于构筑锂离子电池组合电解液}

除此之外, MOFs 材料同样被当作高效的离子篮来 分隔两种不同性质的电解液体系, 使得在同一个电池 内, 实现醚类电解液/离子液体共存的组合电解液的电 池结构设计 ${ }^{[39-40]}$. 例如, Qiao 等 ${ }^{[4]}$ 在 2018 年用 CuBTC $\mathrm{MOF}$ 来隔开 $\mathrm{LiTFSI} / \mathrm{G}_{3}$ (lithium bis(trifluoromethanesulfonyl)imide/triglyme) 和 $\mathrm{Li} / \mathrm{Pyr}_{13}$ TFSI ( $N$-propyl $N$ methylpyrrolidinium bis(trifluoromethanesulfonyl)imide) 电解液 (图 2a 和 2b). 得益于 CuBTC MOF 的微孔结构, 两组电解液的相互穿梭被有效抑制, 进而成功构筑了功 能性组合电解液。在与 $\mathrm{LiNi}_{0.5} \mathrm{Mn}_{1.5} \mathrm{O}_{2} / /$ Graphite $(\mathrm{LNMO} / / \mathrm{G})$ 组装成电池后, 该组合电解液使得 $\mathrm{LNMO} / / \mathrm{G}$ 全电池得以成功且高效地运行 (高温性能优 异, 如图 2c).

\subsection{MOFs 材料用于负极保护}

锂金属由于具有最高的理论比容量 $\left(3860 \mathrm{mAh} \cdot \mathrm{g}^{-1}\right)$ 和最负的电位 (3.040 V, 与标准氢电极相比), 因此被视 为最有前景的负极材料 ${ }^{[42]}$. 但是, 实际运用锂金属负极 面临一系列长期的挑战: 例如, 锂在循环过程中会形成 不均匀的锂枝晶, 最终导致灾难性的电池短路问题和严 重的安全隐患; 锂负极的不断消耗造成电池的库伦效率 低, 进而最终影响电池的容量和使用寿命 ${ }^{[43-45]}$. 抑制锂 枝晶的不均匀生长会大大缓解这一问题. 为了解决这一 难题, Bai 等 ${ }^{[46]}$ 在 2018 年通过 MOF (CuBTC) 的孔道, 对 锂离子的传输进行调控, 获得表面均匀沉积的锂金属电 极(图 3a). 通过将 TFSI-阴离子优先限制在 CuBTC MOF 的亚纳米级的孔道内, 使锂离子得以在 MOF 孔内顺利 传导. MOF 均匀的孔道结构促使通过其中的锂离子能够 均匀地沉积在锂金属表面(图 3b). 锂//锂对称电池显示, 使用 MOF 保护层的电池在 $10 \mathrm{~mA} \cdot \mathrm{cm}^{-2}$ 高电流密度下, 能够维持稳定的曲线, 较小的极化电压 $(120 \mathrm{mV})$ 和稳定 的长循环 $(800 \mathrm{~h})$, 大大优于使用常规电解液的锂//锂

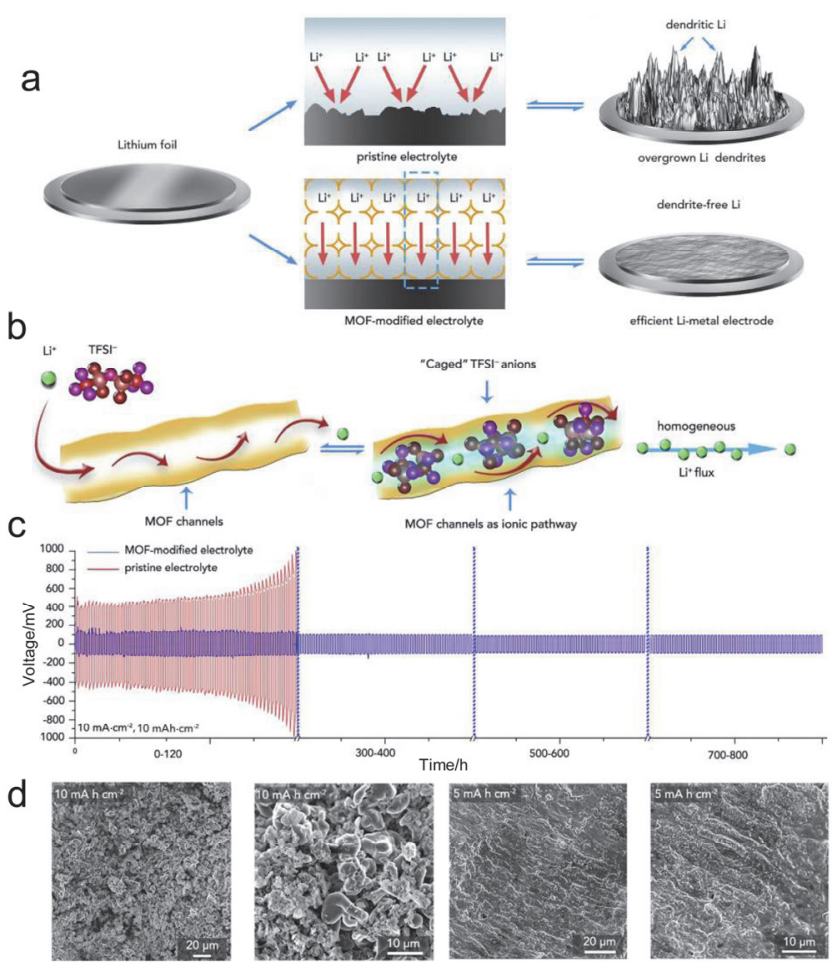

图 3 (a)锂枝晶的生长示意图: 使用原始电解质的循环后的锂金属电 极的示意图(上图); 使用 MOF 保护层的循环后锂金属电极示意图(下 图). (b)锂离子在 MOF 孔道内传导的示意图. (c)使用常规电解液和 MOF 保护层的锂//锂对称电池的电化学性能对比以及(d)对应的循环 后的电极形貌图对比 ${ }^{[46]}$. 经参考文献[46] 许可转载, 版权 2018 Wiley- $\mathrm{VCH}$.

Figure 3 (a) Schematic diagram of the growth of lithium dendrites: a schematic diagram of a lithium metal electrode after cycling using the original electrolyte (top); a schematic diagram of a lithium metal electrode after cycling with a MOF protective layer (bottom). (b) Schematic diagram of lithium ion conduction inside MOF channels. (c) Comparison of electrochemical performance of $\mathrm{Li} / / \mathrm{Li}$ symmetric batteries using conventional electrolyte and MOF protective layer and (d) comparison of the corresponding electrode morphologies after cycling ${ }^{[46] .}$ Reprinted with permission from ref. [46], copyright 2018 Wiley- VCH.

对称电池 (300 h 寿命, $500 \mathrm{mV}$ 极化). 对应的锂金属扫描 电子显微镜结果显示, 使用 MOF 保护层的锂金属表面 较为光滑, 没有明显的锂枝晶. 使用常规电解液的锂金 
属表面形成严重的锂枝晶. 所得结果表明, MOF 在调节 锂金属枝晶方面具有重要意义.

\section{$2.4 \mathrm{MOFs}$ 材料作为准固态电解质}

由于具有丰富的孔道结构, 科研工作者还将 MOFs 材料用作电解液载体, 在 MOFs 材料孔道内填充液态电 解液, 然后将其作为准固态电解质, 改善锂离子电池的 性能. Wang 等 ${ }^{[47]}$ 在 2018 年, 将离子液体(lithium-ionic liquid, Li-IL)填入到多孔 MOF (UiO-67)框架内, 并将其 作为辅助锂离子导体, 用以改善固态电池中由于不稳定 的固体接触而产生的较大的界面电阻 (图 4a). 当与 LLZO $\left(\mathrm{Li}_{7} \mathrm{La}_{3} \mathrm{Zr}_{2} \mathrm{O}_{12}\right)$ 固态电解质 (SSE) 组合后, 固化的 Li-IL 可以通过 MOF 框架的开放通道与 LLZO 颗粒直接 接触, 从而将原本不稳定的固-固接触变为稳定的“纳米 润湿” 界面, 从而增强锂离子的传导. 得益于独特的 “纳 米润湿” 界面, 混合 SSE 表现出高的锂离子电导率(1.0 $\times 10^{-4} \mathrm{~S} \cdot \mathrm{cm}^{-1}$ ) 和 $5.2 \mathrm{~V}$ 的宽电化学窗口, 并且与锂金属 负极具有良好的兼容性. 将所得的准固态电解质和钴酸 锂及 $\mathrm{Li}$ 配对组装成电池后, 电池展示出高的容量保持 率和良好的循环寿命(在 150 次循环仍然能够维持 $97 \%$ 的高容量保持率, 图 4b). Shen 等 ${ }^{[48]}$ 受仿生离子通道的 启发, 将液态电解液 $\left(\mathrm{LiClO}_{4}\right.$-propylene carbonate, $\mathrm{LiClO}_{4}-\mathrm{PC}$ )填入 $\mathrm{MOF}$ 孔道后, 液态电解液的阴离子配 位到 MOF 材料的裸露的金属位点, 使得原本作为锂离
子不良导体的金属有机框架(MOF)转化为高效的锂离 子导体. 直接将其作为准固态电解质 (图 4c 和 $4 \mathrm{~d}$ ), 实现 了快速且高效的锂离子传导过程. 在和 $\mathrm{LiFePO}_{4} / / \mathrm{Li}$ 组 装成电池后，展现出优异的电化学性能(500 圈, 75\%的 容量保持率, 图 4e).

\subsection{MOFs 材料用于调节电解液构型}

近期, Chang 等 ${ }^{[49]}$ 通过在电极表面构筑一层电绝缘 的 MOF 层(ZIF-7), 将有机醚类电解液(LiTFSI-1,3dioxolane/dimethyl ether, LiTFSI-DOL/DME)的电化学窗 口提高到 $4.5 \mathrm{~V}$ (图 5f). 在电池进行充放电过程中, ZIF-7 的狭窄孔道 $(0.29 \mathrm{~nm})$ 可以使溶剂化的锂离子提前去溶 剂化, 锂离子与溶剂之间的相互作用被 MOF 的小孔窗 口阻断, 最终获得了特殊的 “ $\mathrm{Li}^{+}$去溶剂化的醚基电解 液”. 同时, 脱去的溶剂分子, 以一种非活性的“冷冻状” 存在于 MOF 孔内, 去溶剂化后的锂离子与 TFSI 阴离子 构成类盐晶体(图 $5 \mathrm{~b} \sim 5 \mathrm{e}$ ). 得益于特殊的电解液构型, 使用 “ $\mathrm{L} \mathrm{i}{ }^{+}$去溶剂化的醚基电解液” 的 $\mathrm{LiNi}_{0.8} \mathrm{Co}_{0.1} \mathrm{Mn}_{0.1} \mathrm{O}_{2} / / \mathrm{Li}(\mathrm{NCM}-811 / / \mathrm{Li})$ 全电池在有限金属 锂(两倍过量的 Li)的情况下, 保持较好的电化学循环稳 定性, 并且全电池的能量密度能够达到 $630 \mathrm{Wh} \cdot \mathrm{kg}^{-1}$ (仅 考虑 NCM-811 正极和锂负极的重量)(图 5g). 除此之外, Chang 等 ${ }^{[50]}$ 近期又通过选择具有适当孔径的 MOF 材料 (poly(styrenesulfonate), PSS 聚合物修饰的 CuBTC MOF,
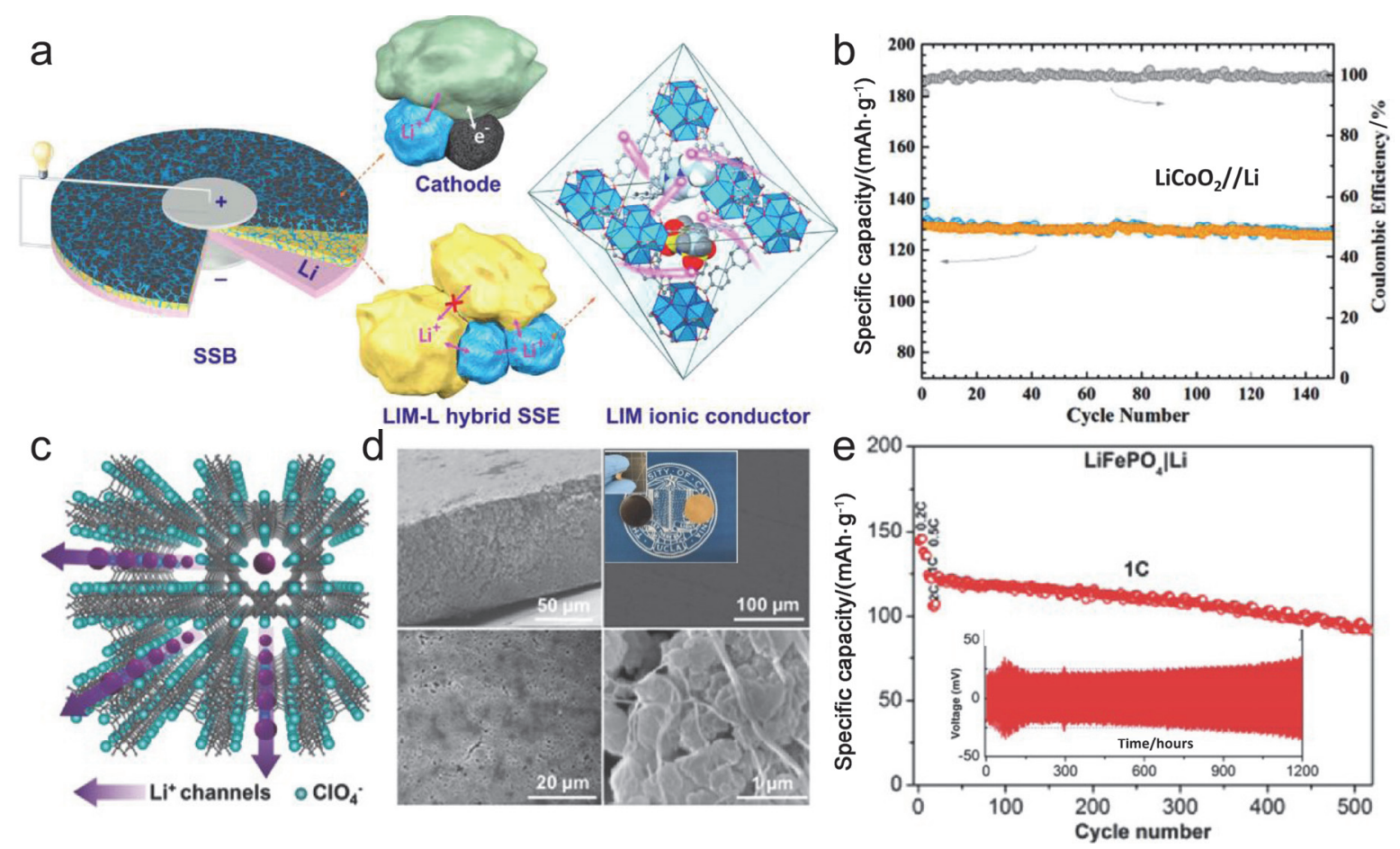

图 4 金属有机框架作为准固态电解质提升锂金属电池的电化学性能. (a, b) UiO-67 MOF 作为辅助锂离子导体, 提高 $\mathrm{LiCoO}_{2} / / \mathrm{Li}$ 电池电化学性 能 ${ }^{[47]}$. 经参考文献[47]许可转载, 版权 2018 Elsevier. (c e) MOF 在被填入部分液态电解液后作为准固态电解质用于 $\mathrm{LiFePO}_{4} / / \mathrm{Li}$ 电池 ${ }^{[48]}$. 经参考 文献[48]许可转载, 版权 2018 Wiley-VCH.

Figure 4 The metal organic framework serves as a quasi-solid electrolyte to improve the electrochemical performance of lithium metal batteries. (a, b) UiO-67 MOF serves as a lithium ion conductor to improve the electrochemical performance of $\mathrm{LiCoO}_{2} / / \mathrm{Li}_{\text {batteries }}{ }^{[47]}$. Reprinted with permission from ref. [47], copyright 2018 Elsevier. (c $\sim \mathrm{e}) \mathrm{MOF}$ is used as a quasi-solid electrolyte for $\mathrm{LiFePO}_{4} / / \mathrm{Li}$ batteries after being filled with part of the liquid electrolyte ${ }^{[48]}$. Reprinted with permission from ref. [48], copyright 2018 Wiley-VCH. 

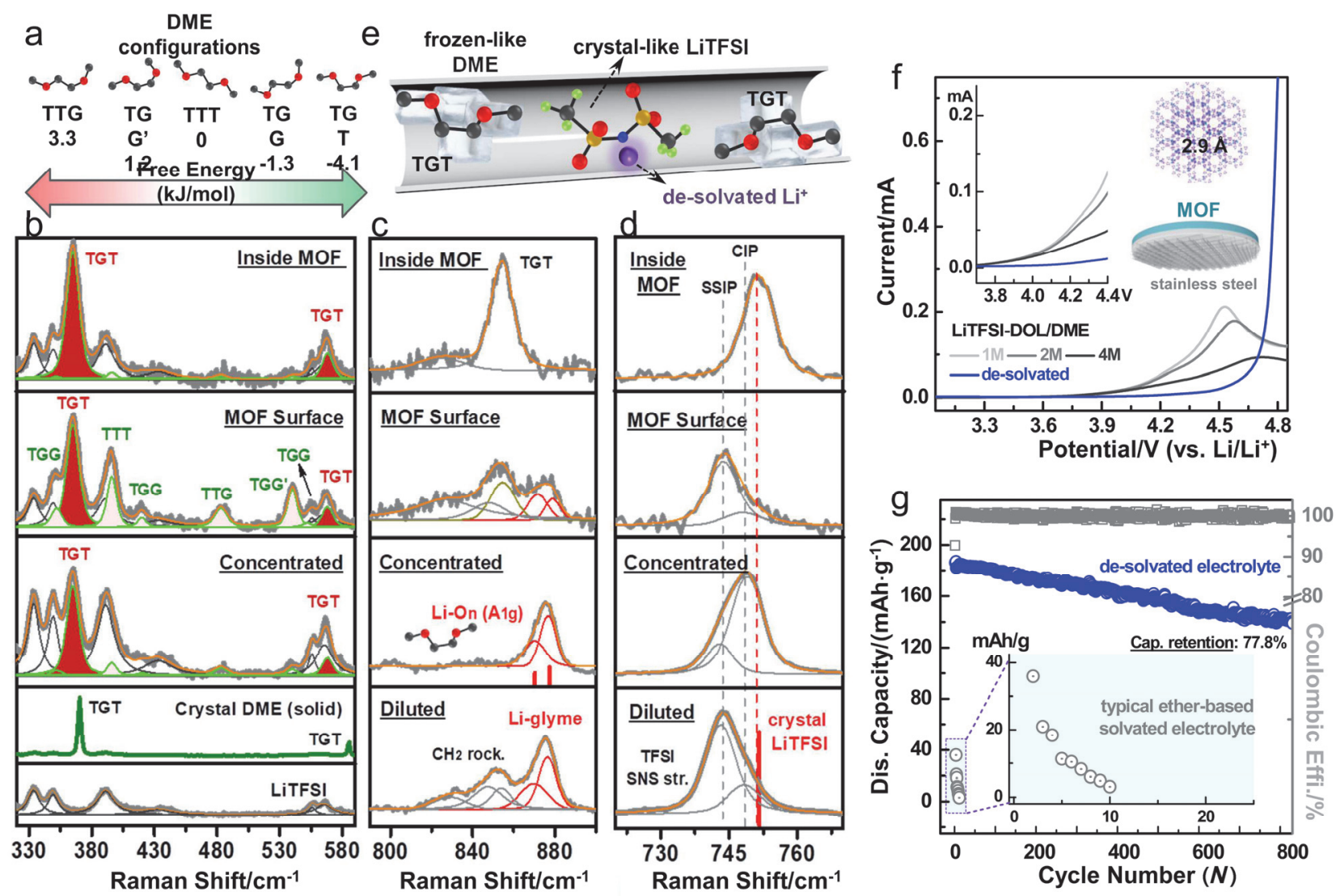

图 5 MOF (ZIF-7)孔道内电解液的拉曼光谱. (a) DME 分子的不同构型及其稳定性示意图. 不同情况下, (b) DME 分子、 (c) Li-DME 相互作用以及 (d) $\mathrm{TFSI}^{-}$对应的拉曼光谱结果. (e)根据实验结果绘制的 MOF 孔内电解液的结构示意图. (f)各电解液的线性扫描伏安(LSV)曲线结果. (g) 使用 “ $\mathrm{Li}^{+}$去溶剂化电解液(醚类)” 的有限金属锂(两倍过量的 $\mathrm{Li}$ )和 NCM-811 配对的 NCM-811//Li 全电池的电化学循环性能 ${ }^{[49]}$. 经参考文献[49]许可转 载, 版权 2020 Cell Press.

Figure 5 Raman spectrum of electrolyte in MOF (ZIF-7) channel. (a) A schematic diagram of DME molecules with different configurations and the corresponding stability under different circumstances, and Raman spectra of (b) DME molecules, (c) Li-DME interaction and (d) TFSI ${ }^{-}$. (e) A schematic diagram of the structure of the electrolyte in the MOF hole drawn according to the experimental results. (f) Linear sweep volt-ampere (LSV) curve results of each electrolyte. (g) The electrochemical cycling performance of NCM-811//Li full-cell based on limited metal lithium (two times excess Li) using "Li ${ }^{+}$ desolvated ether-based electrolyte" ${ }^{\text {"[49] }}$. Reprinted with permission from ref. [49], copyright 2020 Cell Press.

PSS-CuBTC)和电解液体系(PC-LiTFSI), MOF 材料的孔 径效应, 使得 $\mathrm{Li}^{+}$溶剂化壳层之中参与溶剂化的溶剂分 子进一步减少, 获得了一种新型电解液. 该电解液具有 比饱和电解液的构型更加浓缩的电解液构型，同时又不 含任何游离态的溶剂分子. 该电解液具有较宽的电化学 稳定窗口(高于 $5.4 \mathrm{~V}$ vs. $\mathrm{Li}^{-} \mathrm{Li}^{+}$) 和抗氧化能力. 使用该 电解液的 NCM-811//Li 电池表现出稳定的电化学性能. 以上所得结果表明, MOFs 材料在作为准固态电解质的 基底材料方面，具有良好的前景.

\section{3 总结与展望}

如上文所述, 为了使得锂离子电池/锂金属电池的 能量密度、循环寿命以及安全性得到提升, 全世界范围 内的广大科研工作者在诸如开发功能性电解液/添加剂/ 锂盐方面、人造负极保护层以及开发具有高能量密度的 高压正极材料等方面开展了一系列原创性的工作. 近年 来, 科学家们开始使用金属有机框架材料(MOFs)来改
善锂离子电池/锂金属电池中存在的一系列问题，以期 提高电池的容量、循环寿命以及安全性. 通过将 MOFs 材料作为离子篮(抑制锂硫电池的多硫化锂穿梭效应、 分割两种不同电解液构筑组合电解液体系)、负极保护 层(保护锂金属)、准固态电解质(减少液态电解液的使用 量, 提高电池的能量密度)、功能层调控电解液的构型 (使得电解液比高浓电解液的构型更加浓缩, 减少电池 的副反应), 电池的性能得到较大改善. 这些结果得益 于不同的 MOFs 材料的特殊的孔道结构和表界面性质, 使得锂离子电池/锂金属电池用电解液的结构发生了较 大改变, 经过 MOFs 材料孔道调节后的电解液展现出了 特殊的理化性质, 并最终提高了锂离子电池/锂金属电 池的电化学稳定性. 但是, 抛开现阶段所取得的令人惊 喜的各种结果, 目前 MOFs 材料在锂离子电池/锂金属 电池用电解液中的进一步应用尚存在一些问题. 在将 MOFs 材料进一步应用于锂离子电池/锂金属电池之前, 尚需考虑以下三点主要问题: (1) MOFs 的成本问题. 现 
阶段, 虽然通过 MOFs 材料引入到锂离子电池/锂金属 电池可以大大提高电池的容量、寿命以及安全性能, 但 是在当前情况下, MOFs 的生产成本较高, 这无疑会增 加锂离子电池的生产成本. 通过改善 MOFs 的合成工艺, 提高产量, 以及减少其在锂离子电池中的用量, 有望一 定程度地解决成本问题. 例如, 将 MOFs 材料直接涂布 在商业化隔膜上(类似于将 $\mathrm{Al}_{2} \mathrm{O}_{3}$ 涂布在聚丙烯(PP) 和聚 乙烯(PE)隔膜上) 可以大大减少 MOFs 材料在电池体系 中的使用量, 从而进一步降低生产成本. (2) MOFs 材料 在锂离子电池中的化学/电化学稳定性. 大部分 MOFs 材料在有机系锂离子电池/锂金属电池体系中的稳定性 尚需加强. 许多 MOFs 材料对有机电解液不稳定, 因此 不能被用于锂离子电池/锂金属电池体系内. 一部分 MOFs 材料对活泼的金属锂不稳定, 会与其发生化学/电 化学反应. 通过适当的修饰或表面处理, 有望进一步拓 宽 MOFs 材料在锂离子电池/锂金属电池中的应用. (3) MOFs 材料的孔结构/高比表面积利用率. MOFs 材料的 高比表面积决定了很难充分利用其丰富的孔道结构. 选 择合适的溶剂和锂盐, 可以大大提高 MOFs 材料的有效 利用率. 基于 MOFs 材料现阶段在锂离子电池/锂金属 电池体系中已有的诸多优点, 如能有效地解决上述三个 问题, MOFs 材料在锂离子电池/锂金属电池的应用将有 望被进一步拓展.

\section{作者简介}

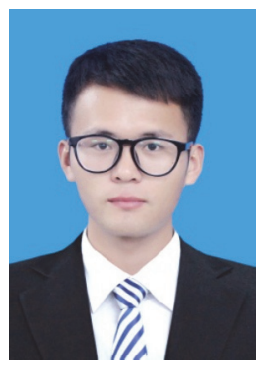

常智, 2017 年 4 月于南京航空航天大学材料科学与技术学 院取得硕士学位. 2020 年 9 月于日本国立筑波大学和日本产业 技术综合研究所(AIST)取得博士学位. 主要研究方向为高比 能锂金属电池体系研发, 具体涉及: 锂一硫电池、锂金属负极 以及相应的电解液/质体系.

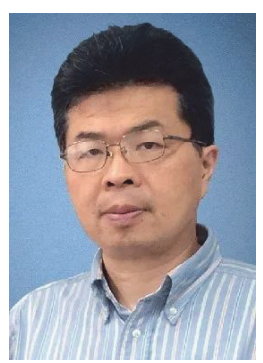

周豪慎, 1985 年获南京大学物理系学士学位, 1994 年获日 本国立东京大学博士学位. 现任南京大学现代工程与应用科 学学院教授, 长江学者. 兼任日本国立产业技术综合研究所 (AIST) 首席研究员、日本国立筑波大学教授. 兼任 Science
Bulletin 常务副主编, Energy Storage Materials 副主编. 长期从 事能源材料、气体传感器、太阳能电池、超级电容器、二次 电池、锂离子电池、锂硫电池、锂空气电池、下一代储能器 件等的研究和开发.

\section{References}

[1] Armand, M.; Tarascon, J. M. Nature 2008, 451, 652.

[2] Dunn, B.; Kamath, H.; Tarascon, J. M. Science 2011, 334, 928.

[3] Tarascon, J. M.; Armand, M. Nature 2001, 414, 359.

[4] Goodenough, J. B.; Kim, Y. Chem. Mater. 2010, 22, 587.

[5] Li, M.; Lu, J.; Chen, Z.; Amine, K. Adv. Mater. 2018, e1800561.

[6] Fan, X.; Chen, L.; Ji, X.; Deng, T.; Hou, S.; Chen, J.; Zheng, J.; Wang, F.; Jiang, J.; Xu, K.; Wang, C. Chem 2018, 4, 174.

[7] Suo, L.; Borodin, O.; Gao, T.; Olguin, M.; Ho, J.; Fan, X.; Luo, C.; Wang, C.; Xu, K. Science 2015, 350, 938.

[8] Jiao, S.; Ren, X.; Cao, R.; Engelhard, M. H.; Liu, Y.; Hu, D.; Mei, D.; Zheng, J.; Zhao, W.; Li, Q.; Liu, N.; Adams, B. D.; Ma, C.; Liu, J.; Zhang, J.-G.; Xu, W. Nat. Energy 2018, 3, 739.

[9] Yamada, Y.; Usui, K.; Sodeyama, K.; Ko, S.; Tateyama, Y.; Yamada, A. Nat. Energy 2016, 1, 1 .

[10] Basile, A.; Bhatt, A. I.; O'Mullane, A. P. Nat. Commun. 2016, 7, ncomms 11794.

[11] Chen, S.; Zheng, J.; Yu, L.; Ren, X.; Engelhard, M. H.; Niu, C.; Lee, H.; Xu, W.; Xiao, J.; Liu, J.; Zhang, J.-G. Joule 2018, 2, 1548.

[12] Duan, H.; Yin, Y. X.; Shi, Y.; Wang, P. F.; Zhang, X. D.; Yang, C. P.; Shi, J. L.; Wen, R.; Guo, Y. G.; Wan, L. J. J. Am. Chem. Soc. 2018 , $140,82$.

[13] Liu, Q. C.; Xu, J. J.; Yuan, S.; Chang, Z. W.; Xu, D.; Yin, Y. B.; Li, L.; Zhong, H. X.; Jiang, Y. S.; Yan, J. M.; Zhang, X. B. Adv. Mater. 2015, 27, 5241 .

[14] Chen, L.; Fan, X.; Hu, E.; Ji, X.; Chen, J.; Hou, S.; Deng, T.; Li, J.; Su, D., Yang, X.; Wang, C. Chem 2019, 5, 896.

[15] Banerjee, R.; Phan, A.; Wang, B.; Knobler, C.; Furukawa, H.; O'Keeffe, M.; Yaghi, O. M. Science 2008, 319, 939.

[16] Kitagawa, S. Chem. Soc. Rev. 2014, 43, 5415.

[17] Zhang, J.; Li, P.; Zhang, X.; Ma, X.; Wang, B. Acta Chim. Sinica 2020, 78, 597. (张晋维, 李平, 张馨凝, 马小杰, 王博, 化学学报, 2020, 78, 597.)

[18] Qi, Y.; Ren, S.; Che, Y.; Ye, J.; Ning, G. Acta Chim. Sinica 2020, 78, 613. (齐野, 任双颂, 车颖, 叶俊伟, 宁桂玲, 化学学报, 2020, 78, 613.)

[19] Guo, Z.; Zhang, Y.; Feng, X. Acta Chim. Sinica 2020, 78, 397. (郭振 涁, 张媛媛, 冯霄, 化学学报, 2020, 78, 397.)

[20] Cai, C.; Li, L.; Deng, X.; Li, S.; Liang, H.; Qiao, Z. Acta Chim. Sinica 2020,78, 427. (蔡铖智, 李丽风, 邓小梅, 李树华, 梁红, 乔智威, 化学学报, 2020, 78, 427.)

[21] Hou, Q.; Wu, Y.; Zhou, S.; Wei, Y.; Caro, J.; Wang, H. Angew. Chem. Int. Ed. 2019, 58, 327.

[22] Li, Z.; Li, H.; Guan, X.; Tang, J.; Yusran, Y.; Li, Z.; Xue, M.; Fang, Q.; Yan, Y.; Valtchev, V.; Qiu, S. J. Am. Chem. Soc. 2017, 139, 17771.

[23] Rodenas, T.; Luz, I.; Prieto, G.; Seoane, B.; Miro, H.; Corma, A.; Kapteijn, F.; Llabres, I.X.F.X.; Gascon, J. Nat. Mater. 2015, 14, 48.

[24] Wang, H.-F.; Chen, L.; Pang, H.; Kaskel, S.; Xu, Q. Chem. Soc. Rev. 2020, 49, 1414.

[25] Hong, W.; Kitta, M.; Xu, Q. Small Methods 2018, 2, 1800214.

[26] Ji, S.; Chen, Y.; Zhao, S.; Chen, W.; Shi, L.; Wang, Y.; Dong, J.; Li, Z.; Li, F.; Chen, C.; Peng, Q.; Li, J.; Wang, D.; Li, Y. Angew. Chem. Int. Ed. 2019, 58, 4271.

[27] Miner, E. M.; Park, S. S.; Dinca, M. J. Am. Chem. Soc. 2019, 141, 4422.

[28] Shen, L.; Wu, H. B.; Liu, F.; Brosmer, J. L.; Shen, G.; Wang, X.; Zink, J. I.; Xiao, Q.; Cai, M.; Wang, G.; Lu, Y.; Dunn, B. Adv. Mater. 2018, 30, e1707476.

[29] Bruce, P. G.; Freunberger, S. A.; Hardwick, L. J.; Tarascon, J.-M. Nat. Mater. 2012, 11, 19.

[30] Chang, Z.; Ding, B.; Dou, H.; Wang, J.; Xu, G.; Zhang, X. Chem.-Eur. J. 2018, 24, 3768.

[31] Chang, Z.; He, Y.; Deng, H.; Li, X.; Wu, S.; Qiao, Y.; Wang, P.; Zhou, H. Adv. Funct. Mater. 2018, 28, 1804777.

[32] Chang, Z.; Qiao, Y.; Wang, J.; Deng, H.; He, P.; Zhou, H. Energy Storage Mater. 2020, 25, 164. 
[33] Ding, B.; Wang, J.; Chang, Z.; Xu, G.; Hao, X.; Shen, L.; Dou, H.; Zhang, X. ChemElectroChem 2016, 3, 668.

[34] Ji, X.; Lee, K. T.; Nazar, L. F. Nat. Mater. 2009, 8, 500.

[35] Li, Y.-J.; Fan, J.-M.; Zheng, M.-S.; Dong, Q.-F. Energy Environ. Sci. 2016, 9, 1998.

[36] Wang, J.; Chang, Z.; Ding, B.; Li, T.; Yang, G.; Pang, Z.; Nakato, T.; Eguchi, M.; Kang, Y. M.; Na, J. Guan, B.; Yamauchi, Y. Angew. Chem. Int. Ed. 2020, 59, 19570.

[37] Bai, S.; Liu, X.; Zhu, K.; Wu, S.; Zhou, H. Nat. Energy 2016, 1, 16094.

[38] Zang, Y.; Pei, F.; Huang, J.; Fu, Z.; Xu, G.; Fang, X. Adv. Energy Mater. 2018, 8, 1870136.

[39] Qiao, Y.; He, Y.; Wu, S.; Jiang, K.; Li, X.; Guo, S.; He, P.; Zhou, H. ACS Energy Lett. 2018, 3, 463.

[40] Qiao, Y.; Jiang, K.; Li, X.; Deng, H.; He, Y.; Chang, Z.; Wu, S.; Guo, S.; Zhou, H. Adv. Energy Mater. 2018, 8, 1801120.

[41] Qiao, Y.; He, Y.; Jiang, K.; Liu, Y.; Li, X.; Jia, M.; Guo, S.; Zhou, H. Adv. Energy Mater. 2018, 8, 1802322

[42] Chang, Z.; Qiao, Y.; Deng, H.; Yang, H.; He, P.; Zhou, H. Energy Environ. Sci. 2020, 13, 1197.

[43] Chen, S.; Zheng, J.; Yu, L.; Ren, X.; Engelhard, M. H.; Niu, C.; Lee,
H.; Xu, W.; Xiao, J.; Liu, J. Joule 2018, 2, 1548.

[44] Fan, X.; Chen, L.; Borodin, O.; Ji, X.; Chen, J.; Hou, S.; Deng, T.; Zheng, J.; Yang, C.; Liou, S. -C.; Amine, K.; Xu K.; Wang, C. Nat. Nanotech. 2018, 13, 715 .

[45] Liu, J.; Bao, Z.; Cui, Y.; Dufek, E. J.; Goodenough, J. B.; Khalifah, P.; Li, Q.; Liaw, B. Y.; Liu, P.; Manthiram, A.; Meng, Y. S.; Subramanian, V. R.; Toney, M. F.; Viswanathan, V. V.; Whittingham, M. S.; Xiao, J.; Xu, W.; Yang, J.; Yang, X.-Q.; Zhang, J.-G. Nat. Energy 2019, 4, 180 .

[46] Bai, S.; Sun, Y.; Yi, J.; He, Y.; Qiao, Y.; Zhou, H. Joule 2018, 2, 2117.

[47] Wang, Z.; Wang, Z.; Yang, L.; Wang, H.; Song, Y.; Han, L.; Yang, K.; Hu, J.; Chen, H.; Pan, F. Nano Energy 2018, 49, 580.

[48] Shen, L.; Wu, H. B.; Liu, F.; Brosmer, J. L.; Shen, G.; Wang, X.; Zink, J. I.; Xiao, Q.; Cai, M.; Wang, G. Adv. Mater. 2018, 30, 1707476.

[49] Chang, Z.; Qiao, Y.; Deng, H.; Yang, H.; He, P.; Zhou, H. Joule 2020, 4, 1776.

[50] Chang, Z.; Qiao, Y.; Yang, H.; Deng, H.; Zhu, X.; He, P.; Zhou, H. Energy Environ. Sci. 2020, 13, 4122.

(Cheng, B.) 